Revista Electrónica Teoría de la Educación.

Educación y Cultura en la Sociedad de la Información.

http://www.usal.es/teoriaeducacion

Vol. 7. N22. Diciembre 2006

\title{
LOS NUEVOS CÓDIGOS DE LA COMUNICACIÓN EMOCIONAL UTILIZA- DOS EN INTERNET
}

Como en la conocida frase "el medio es el mensaje" en la cual McLuhan resume categóricamente la primacía de la tecnología de la comunicación en la formación de la civilización, esta autora considera que toda comunicación, independientemente del medio que utilice, debe ser eficaz per se, aunque existan adaptaciones de este mensaje a cada respectivo medio. McLuhan estaba en lo cierto en el sentido que cada medio trasmite un mensaje diferenciado, podría decirse por tanto que, mismo fondo pero diferente forma, y este artículo pretende incursionar en ambos, tanto en el fondo, entendiéndose por ello el aspecto emocional de la comunicación en la CMO, como su forma, analizando el lenguaje utilizado para trasmitirla.

Palabras clave: Comunicación Emocional, CMO, Lenguaje, Códigos.

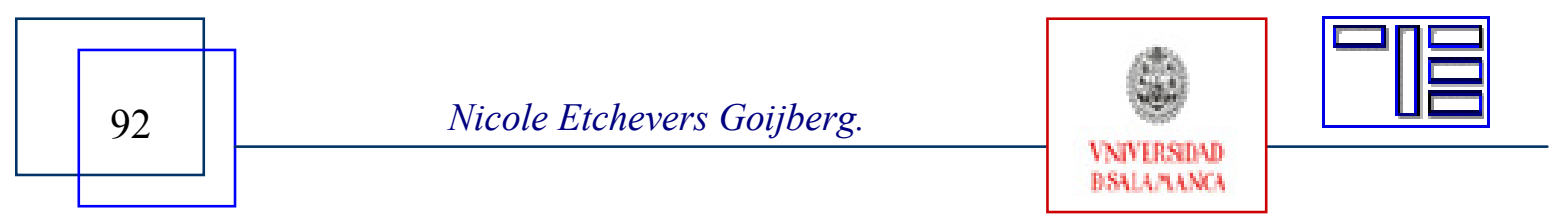




\section{NEW CODES OF EMOTIONAL COMMUNICATION IN THE INTERNET}

As in the well-known phrase "the medium is the message" in which McLuhan categorically summarizes the importance of the communication's technology in the formation of civilization, this author considers that all communication, independently of the medium used, must be effective by itself, even if adaptations of this message are required for each respective medium. McLuhan was in the certain in the sense that each medium offers a differentiated message, could be said though, same bottom but different form, and this article tries to reach in both, at the core, being this understood as the emotional aspect of the communication in the $\mathrm{CMC}$, as in its form, analysing the language to transmit it.

Key words: Emotional Communication, CMC, Language, Codes.

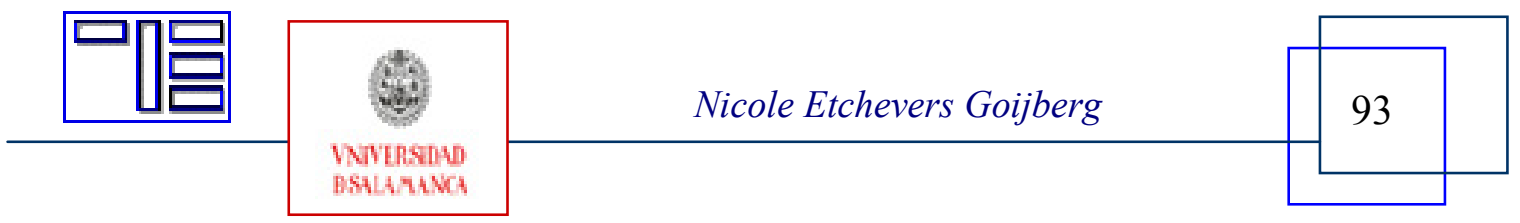




\section{LES NOUVEAUX CODES DE LA COMMUNICATION EMOTIONNELLLE UTILISÉS SUR INTERNET}

Comme dans la phrase connue "Le média est le message" dans laquelle McLuhan résume de manière catégorique la suprématie de la technologie de la communication comme la formation de la civilisation, cette auteur considère que toute communication, indépendamment du média quelle utilise, doit éter efficace, bien qu'il existe des adaptations de ce message pour chaque média respectif. McLuhan était dans le vrai, dans le sens ou chaque média transmet un message différent, pourtant on pourrait se dire que, le fond est le

même mais la forme est différente, et cet article pourrait s'introduire (dans chaque), tant dans le fond, entendant de ce fait l'aspect émotionnel de la communication dans la $\mathrm{CMO}$, que dans la forme, analysant le langage utilisé pour la transmettre.

Mots clefs: Communication émotionnelle, CMO, Langage, Codes.

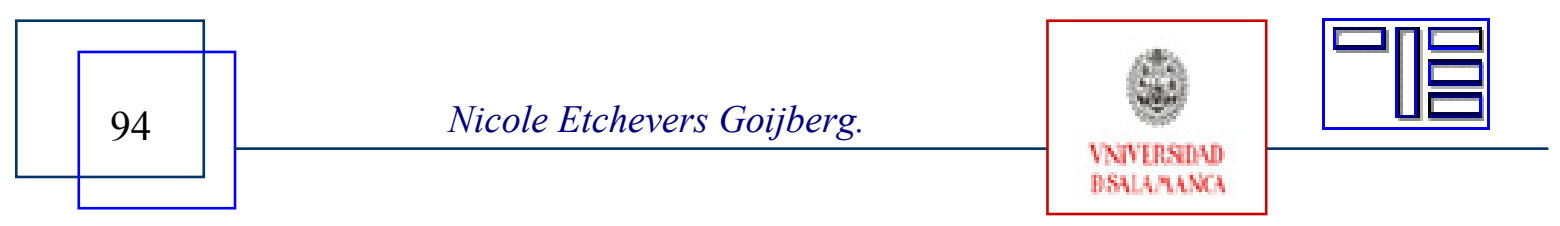




\section{LOS NUEVOS CÓDIGOS DE LA COMUNICACIÓN EMOCIONAL UTILIZA- DOS EN INTERNET}

Nicole Etchevers Goijberg

nietcheg@hotmail.com

Observatorio para la CiberSociedad (ESPAÑA)

\section{1.- INTRODUCCIÓN}

En los albores del siglo XXI, es innegable el rol fundamental que están desempeñando las comunicaciones en la creación de nuevas perspectivas socio-culturales, de negocios o de relaciones interpersonales, sin que para ello sea una limitación ni las distancias, ni los tiempos, ni los espacios. La investigación de los fenómenos comunicacionales está involucrándose desde hace algún tiempo en torno a estos cambios: en analizar qué sucede con la intromisión de estas tecnologías en nuestra vida diaria; en lo que sucede personal y psicológicamente en cada uno de estos nuevos usuarios y en las nuevas formas de lenguaje creadas para comunicarse a través de estos medios.

Los últimos desarrollos tecnológicos han demostrado, con creces, ofrecer mayores oportunidades para la comunicación entre individuos separados por grandes distancias físicas, así como también para la comunicación dentro de pequeños y más grandes grupos de individuos; entre individuos y grandes cantidades de conocimiento fácilmente accesibles y; entre poderosos centros de comunicación, como grandes organizaciones, compañías y autoridades (Rosengren, 2000).

En otras palabras, se están analizando los nuevos lenguajes y formas de comunicación utilizadas mediante las nuevas Tecnologías de la Información y la Comunicación (TIC). Es así, entonces, que se pretende comprender y descifrar la carga emotiva de estos nuevos lenguajes, es decir, prácticamente siempre, puesto que la comunicación siempre conlleva una carga emocional.

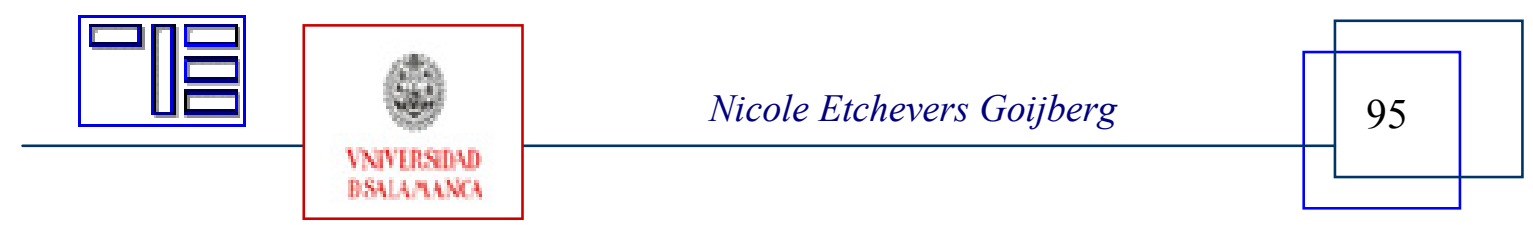


Revista Electrónica Teoría de la Educación.

Educación y Cultura en la Sociedad de la Información.

http://www.usal.es/teoriaeducacion

Vol. 7. No2. Diciembre 2006

\section{2.- COMUNICACIÓN MEDIADA POR ORDENADOR (CMO)}

El email ha sido desde los inicios de Internet -en la década de los 70- la principal herramienta de comunicación online, sin embargo, no fue hasta la década de los 90 cuando se convierte en una herramienta de uso masivo y por ende, cobra significado su investigación (Mayans, 2001). Sin ir más lejos, la Rand Corporation (http://www.rand.org) en su investigación de 1995 sobre el uso del email, lo confirma como la forma de interacción más extendida en el uso de las redes (Smith y Kollock, 2003). De igual modo lo demuestran las conclusiones del último estudio de Red.es (Enero - Marzo 2005) sobre "Uso y Perfil de Usuarios de Internet" en España, en donde se expone que el correo electrónico (email), con un $78,1 \%$, continúa siendo la herramienta de comunicación más usada por los internautas. En este mismo estudio, bastante más abajo se encuentran los índices de Mensajería Instantánea y Chat, con un 44,8\% y $23,5 \%$ respectivamente. Si a lo anterior se le agregan las conclusiones de otras investigaciones en las cuales se define "a la comunicación interpersonal como el uso dominante de Internet" (Boneva, B.; Kraut, R. y Frohlich, D. 2000; Kraut, R., Mukopadhyay, T., Szazypula, J., Kiesler, S., y Scherlis, W. 2000; Pew Internet and American Life Project, 2000b), es relativamente fácil entrever la atracción de esta temática para los investigadores sociales.

Del mismo modo en que se aduce un encanto particular en la escritura del email, existen también normas particulares y representativas que sólo conciernen a esta herramienta de comunicación online. Al igual que en la comunicación no verbal, se podría decir que en la CMO también existen reglas de expresión (display rules) (Scherer y Ekman,1984) que hacen que las expresiones emocionales y las formas de comportarse sean manifestadas y reguladas de formas diferentes por las distintas culturas y grupos online. Estas reglas de comunicación online que rigen la Red son conocidas como netiquette -reglas de etiqueta en la Red o traducidas comúnmente como netiqueta- y surgen entre los usuarios de comunidades en red (por ejemplo, Usenet) a modo de controlar el número de mensajes ofensivos que circulan. Toda vez que el grupo de usuarios de Internet ha crecido desmedidamente, las "reglas de netiqueta" han pasado a formar parte de la cultura intrínseca de este medio, reconocida y aceptada por gran parte de los usuarios, a pesar que pueden existir pequeñas diferencias dependiendo de la cultura y el contexto en el que sean utilizadas.

De la Torre expone que las manifestaciones de la emoción son de naturaleza neurofisiológica, corporal, psicológica y social. Entre las primeras se sitúan las respuestas involuntarias del tipo: rubor, sudoración, taquicardia, presión sanguínea, alteración de la respiración, sequedad bucal, y sobre todo el papel de los neurotransmisores. Estos últimos, al igual que las hormonas se encuentran siempre implicados en todo proceso emocional. Entre las segundas, las respuestas de índole comportamental o corporal -que a veces pueden llegar a disimularse-, están las expresiones faciales, posturas corporales y el paralenguaje. Algunos otros se dan como movimientos inconscientes de reajuste del cuerpo. La comunicación no verbal y el paralenguaje proporcionan variados indicios

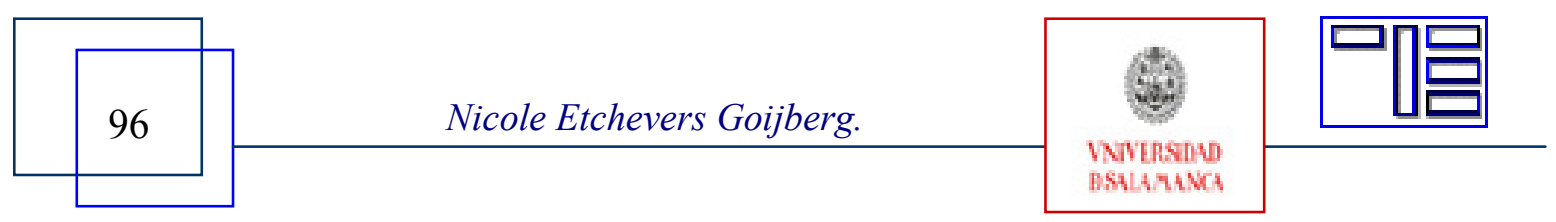


para inferir emociones de ira, rabia, tristeza, alegría, amor, odio, etc. En tercer lugar, el sentimiento, sería la expresión psicológica de la emoción; así, se habla de sentimientos de amor, ternura, afecto, tristeza, etc. Si la emoción es muy fuerte puede provocar fobias, depresión, estrés y otros comportamientos patológicos como obsesiones compulsivas. Estas alteraciones pueden tener su origen en emociones positivas como amor convertido en desamor o el orgullo personal cuando se convierte en superego; o en emociones negativas como cuando los celos generan obsesiones compulsivas. Por último, la expresión social de las emociones, por ejemplo, el orgullo (de la propia cultura, pueblo o raza) que puede dar lugar a la intolerancia cuando se extrema; la filantropía como sentimiento de entrega a los demás o la vergüenza. De hecho muchas de las emociones personales pueden tener su equivalente social como el miedo convertido en pánico colectivo o la aversión convertida en hostilidad.

Para cada una de las manifestaciones descritas: neurofisiológica, corporal, psicológica y social, se puede encontrar alguna representación emocional (expresada de una o de otra manera) en la $\mathrm{CMO}$, ya sea tácitamente por el lenguaje escrito (textual y gráfico) o a través de éste más lo que en esta investigación se denomina "lenguaje complementado".

"Imagino que el lenguaje escrito se transforma en nuestra mente en sensaciones, imágenes, sonidos, olores e incluso en sabores, y en cuanto sucede, dichas imágenes reciben el mismo tratamiento por parte de los mecanismos de evaluación automática que cualquier otro acontecimiento". (Ekman, 2004, 56)

Aunque, por una parte, las palabras facilitan la expresión emocional, éstas no son suficientes para trasmitir la completa trama emocional humana, por tanto, generalmente van acompañadas de algún aspecto de la comunicación no verbal, conciente o inconsciente, para que el mensaje sea trasmitido y recibido eficaz y acertadamente. De este modo, se llega a un punto en donde es imperioso preguntarse ¿de qué manera, entonces, está siendo traducido el aspecto emocional en la comunicación por Internet?, puesto que evidentemente se ha convertido en un medio en donde las emociones fluyen copiosamente (Arango Pinto, 2004; Baker, 2000; Bescós, 2002; Cummings, Butler y Kraut, 2002; Del Brutto, 1999; Donn y Sherman, 2002; Etchevers, 2006; Gil, Feliu, Rivero y Gil, 2003; Gordo López, 2002; Mayans, 2006; McCown, Fischer, Page y Homant, 2001; Roco, 2001). Así, es en las relaciones afectivas online (de amistad o amorosas) donde más se despliega el repertorio emocional, puesto que se intenta abrir o representar el self (yo) lo máximo posible al otro. Aunque existen relaciones interpersonales que no necesariamente se encuentran en esta categoría afectiva de amistad o amor -como las interacciones en un contexto educativo o profesionales-, igualmente, y a pesar que muchos quisieran obviarlo, varias expresiones de este repertorio emocional son también frecuentemente utilizadas. A modo de ejemplo se pueden citar los intentos de "seducir" al compañero de trabajo o al jefe para convencerles de una nueva idea; las sonrisas expresadas cuando estamos seguros y satisfechos de los logros; cuando hay empatía con un compañero de clase o admiración ante el profesor, etc.

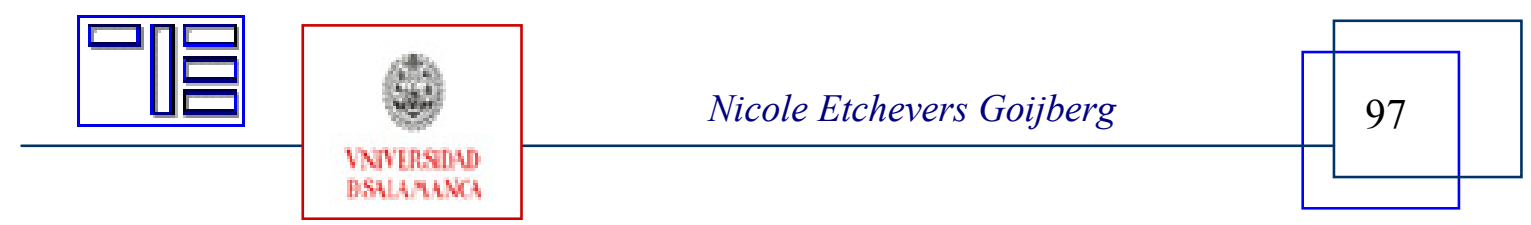


Revista Electrónica Teoría de la Educación.

Educación y Cultura en la Sociedad de la Información.

http://www.usal.es/teoriaeducacion

Vol. 7. No2. Diciembre 2006

\section{3.- NUEVOS CÓDIGOS DE COMUNICACIÓN EMOCIONAL, O LO QUE VENDRÍA A LLAMARSE, EL LENGUAJE COMPLEMENTADO EN LA CMO}

Esta autora trabaja bajo la premisa "El lenguaje complementado es a la comunicación virtual lo que el lenguaje no verbal es a la comunicación presencial". Con ello, se cree que este lenguaje complementado puede relacionarse directamente con los diferentes aspectos emocionales que componen toda comunicación emocional permitiendo deducir información de las características personales y de personalidad de los sujetos comunicantes.

Al utilizar el constructo lenguaje complementado se ha querido inferir que además del lenguaje normal y cotidiano utilizado para comunicarse por escrito en estos medios virtuales, en los últimos años los propios usuarios han creado nuevos códigos que sirven para reforzar o "complementar" la información mediada por simples palabras.

\subsection{Emoticones}

Los emoticones fueron uno de los primeros códigos originales creados por los usuarios de Internet para representar estados emocionales y sentimientos a través de la CMO . Este neologismo del inglés, emoticons, se compone mediante dos palabras: emotion + icon, y sus orígenes se remontan a 1982, cuando Scott Fahlman del departamento de ciencias informáticas de la Universidad Carnegie Mellon, con el objetivo de evitar mal entendidos entres sus colegas a la hora de enviar chistes en sus emails, propuso que las bromas enviadas fuesen marcadas con un signo de caracteres no-alfanuméricos que asemejaban una cara sonriente recostada horizontalmente, más conocida hoy en día como smilie o "carita feliz":)

Desde esa lejana fecha - para la rapidez con que transcurre todo en el ciberespacio- se ha instaurando una gran producción de emoticones que intentan representar emociones y tendencias a la acción de los sujetos comunicantes. Estos emoticones cumplen una función informativa y complementaria a las palabras, como lo hace la comunicación no verbal (kinesia y paralenguaje), y en específico las expresiones faciales en la comunicación cara a cara, pero también trasmiten intencionadamente su propio mensaje o significado. Lo que debe quedar claro, es que los emoticones en ningún caso cumplen una función "sustituyente" del lenguaje corporal, o dicho en otros términos, estos signos textuales emocionales no reemplazan la comunicación no verbal (imposible de sustituir) pero sí complementan y otorgan información anexa y similar a la que se trasmite originalmente por esta vía. "Ante la textualidad y acorporeidad del medio, el emoticon es a la vez el vehículo transmisor y el contenido trasmitido" (Mayans, 2001).

Los emblemas faciales, identificados claramente en los estudios kinésicos (Ekman y Friesen, 1969) también se ven representados en los emoticones, como por ejemplo, "el guiño" ;-), siendo de hecho, junto con la cara feliz :-) y la cara triste :-( , uno de las signos más frecuentes entre los usuarios de Internet. Este guiño virtual, al igual que sucede

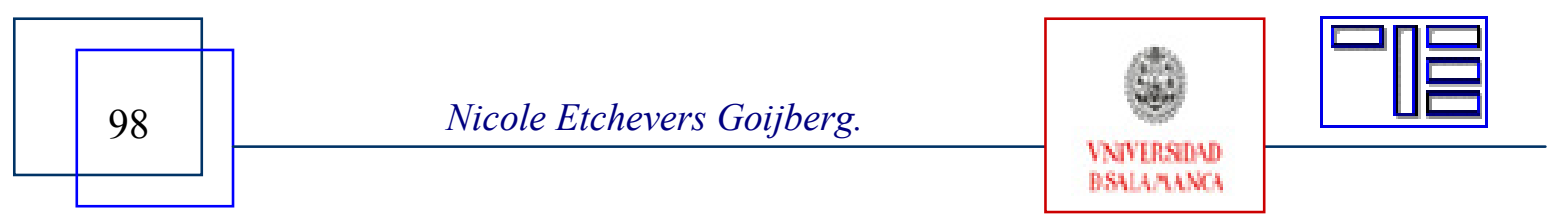


en la comunicación cara a cara, es usado tan deliberadamente como una palabra y su significación puede cambiar dependiendo del contexto (confianza, complicidad, broma, ironía o coqueteo) o de la personalidad de los sujetos interactuantes (risueños, bromistas, sarcásticos, ligones, coquetos, etc.).

Con el avance de la tecnología, las herramientas actuales de CMO ya no están limitadas únicamente a ofrecer simulacros de los gestos humanos mediante los caracteres que el teclado dispone, si no que es posible integrar íconos gráficos estáticos y dinámicos (gifs). De esta manera, si anteriormente era necesario conocer los códigos y utilizar la imaginación para evocar textualmente una emoción dada, con el advenimiento de los íconos, la comunicación emocional se convierte más representativa y menos imaginativa -menos simulada-, pasando a ser de fácil uso y comprensión para la mayoría de los sujetos, a pesar de que su significado siga dependiendo del contexto en el que se vea inserto.

Tomando en cuenta lo anterior, al igual que Ekman con su teoría de las Emociones Básicas Universales, en el ciberespacio existe también una emocionalidad básica universal que se encuentra representada sobre todo en los emoticones de "carita feliz", "carita triste" y "guiño". Si bien es cierto que estos emoticones son los más utilizados en la CMO de todas partes del mundo, existe un largo listado de otros emoticones que corresponden a las diferencias suministradas por los aspectos propios de cada cultura, y en el caso que se estudia, de cada entorno o comunidad virtual. Entre ellas se puede observar no únicamente los referentes a las emociones básicas universales (alegría, tristeza, ira, miedo, aversión y sorpresa), sino otras categorías emocionales, como las emociones sociales (ej. la vergüenza), los gestos emblema (ej. pulgar arriba o abajo indicando aprobación o desaprobación respectivamente), los signos conversacionales (ej. movimientos de ceja para acentuar un discurso) o la utilización de varios íconos gráficos encadenados para representar un estado emocional específico, pareciéndose así a la verdadera "trama" (García Carrasco y García del Dujo, 2001) de acontecimientos, experiencia y elementos externos que componen y son, las emociones.

Queda claro, entonces, que existen los medios para representar y comunicar las emociones, sin embargo, ¿están cumpliendo éstos su función? ¿son realmente eficaces?. A este respecto, en la $7^{\mathrm{a}}$ y última edición de la encuesta a usuarios de Internet "Navegantes en la Red" realizada por la AIMC -Asociación para la Investigación de los Medios de Comunicación (Febrero 2005), en donde se le preguntó a usuarios de la Red si hacían uso de estos elementos, el $62,7 \%$ de los participantes de la encuesta contestaron utilizar emoticones (ver Figura 1).

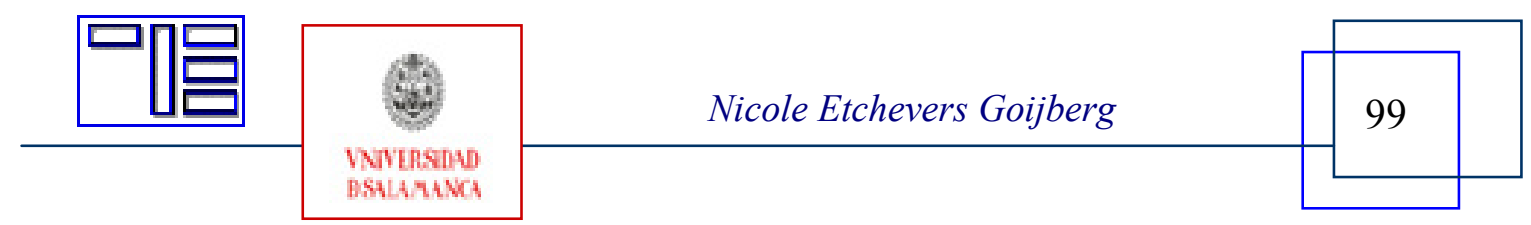


Revista Electrónica Teoría de la Educación.

Educación y Cultura en la Sociedad de la Información.

http://www.usal.es/teoriaeducacion

Vol. 7. No2. Diciembre 2006

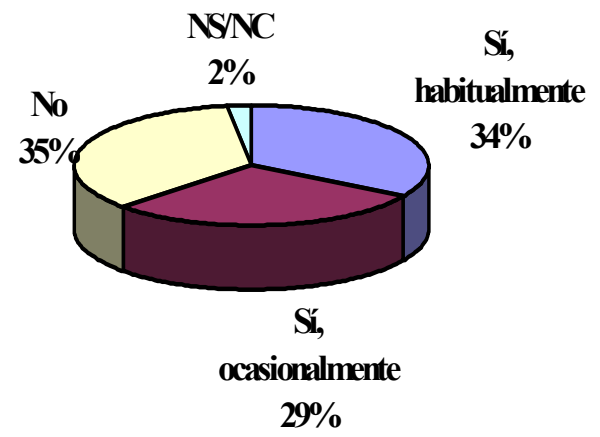

Figura. 1. Uso de Emoticones por los usuarios de Internet

A pesar de que los emoticones no siempre pueden ser utilizados -ni tampoco para todas las ocasiones-, las estadísticas demuestran que una gran cantidad de usuarios de Internet, y en específico los que utilizan este medio para entablar relaciones interpersonales, encuentran los emoticones de gran apoyo y los emplean para ayudarse a comunicar de manera más integral.

\subsection{Alteraciones de las formas lingüísticas}

Así como los emoticones, otros aspectos relacionados al lenguaje se han agregado a los nuevos códigos surgidos del uso de las Tecnologías de la Información y Comunicación (TIC). La gran mayoría de las herramientas comunicacionales utilizadas hoy en día en Internet se encuentran inundadas de errores ortográficos y tipográficos, abreviaciones, contracciones, usos indebidos de reglas gramaticales y puntuación, onomatopeyas y acrónimos. El mencionar estas alteraciones lingüísticas no corresponde al mero hecho de dejar constancia de las trasgresiones efectuadas a las reglas de escritura sino corresponde más bien a que todas ellas se incluyen dentro del denominado lenguaje complementado puesto que implican una carga emocional significativa.

Cada entorno mediado puede utilizar diferentes códigos y reglas de escritura que son de uso exclusivo de ese grupo de usuarios, no obstante, existen algunas alteraciones lingüísticas más representativas utilizadas por gran parte de los usuarios de la CMO, independientemente de la herramienta de comunicación utilizada. Así, tanto en emails, chats y mensajería instantánea es normal encontrar (i) sustitución de consonantes complejas (qu, ll, ch) por simples ( $\mathrm{k}, \mathrm{y}, \mathrm{x}$ ); (ii) abreviación de palabras (que $=\mathrm{k}$, q; por $=\mathrm{x}$; porque = pq, xq); (iii) contracción de palabras eliminando consonantes o sílabas (quedado = kedao; quedamos = kdmos); (iv) uso de mayúsculas (PORFAVOR); (v) repetición de vocablos (exceleeeeeeeeeente); (vi) intensificación y repetición de signos de puntuación (gracias!!!!!); (vii) onomatopeyas (jajajaja, mua) y (viii) acrónimos (lol).

La escasez de oportunidades para expresar emociones de manera no verbal, es uno de los aspectos que caracteriza la CMO textual. De este modo, los usuarios han tenido que

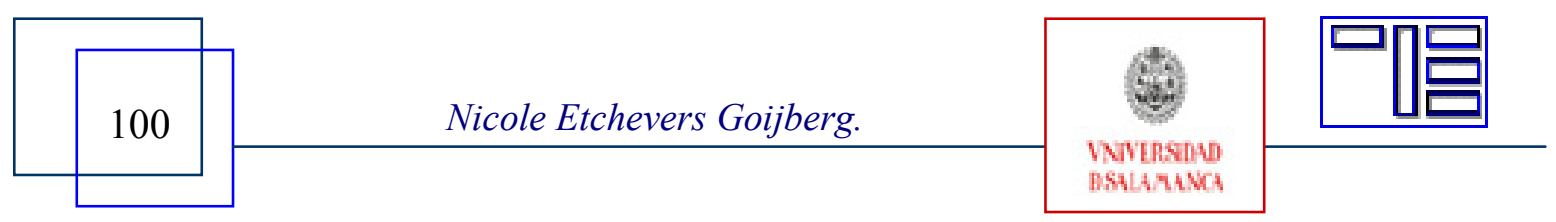


crear (e imaginar) nuevas formas de comunicación -nuevos códigos- en donde sea posible reforzar estas limitaciones. De este modo, si bien es cierto que la CMO textual jamás podrá tener la riqueza paralingüística de la comunicación presencial, puesto que no se puede oír al otro en un entorno meramente textual, se considera que la creatividad con la que ha sido paliada la falta de corporalidad en los medios electrónicos textuales es sorprendente.

Uno de los mejores ejemplos de lo anterior es, sin duda, el uso voluntario de MAYÚSCULAS que complementa al texto escrito para que éste logre trasmitir, aunque sea de manera "simulada", la información paraverbal emocional que generalmente se expone de manera natural en una conversación cara a cara. El hecho de escribir con mayúsculas en la CMO suele indicar un énfasis o reforzamiento que, en el caso de estar frente a otra persona, haría la voz de forma natural. Como se observa en las reglas de etiqueta en la Red (netiquette), en la CMO se ha aceptado de forma prácticamente tácita que las mayúsculas tienden a significar un aumento en el tono de la conversación, o en otras términos, que el sujeto que escribe utilizándolas se encuentra alzando la voz o gritando. Si esta situación se analiza desde la perspectiva de las teorías sobre comunicación no verbal, se puede confirmar uno de los componentes característicos del paralenguaje, a saber, la intensidad o volumen de voz (de muy fuerte a muy suave) de los cualificadores vocales de las vocalizaciones (Trager, 1958 cit. en Knapp, 1982). A su vez, el volumen de voz también se encuentra correlacionado con algunas de las expresiones vocales emocionales (Davitz, 1964); por tanto, si se toma en cuenta que el uso continúo de mayúsculas en la CMO es interpretado como que el sujeto comunicante está "gritando" o está enfatizando sus palabras-, se puede deducir, que las mayúsculas del texto escrito en la CMO representan (simulan) el máximo parámetro de medición, es decir, el equivalente al volumen de voz "alto". Si para Davitz, las emociones de Cólera, Alegría y Jovialidad se expresaban con una característica de volumen de voz de "moderadamente Alto" a "Alto", entonces, en la CMO, estas mismas expresiones emocionales pueden trasmitirse eficazmente mediante el uso de mayúsculas del lenguaje complementado.

Por otra parte, el parámetro de extensión (desde arrastrar las palabras hasta el hablar extremadamente cortado) se ve reflejado en las repeticiones o arrastre de vocablos ya que estos sugieren una clara intención por imitar las inflexiones (firmeza, irregularidad, monotonía, etc.) y elocuciones (ligadas, entrecortadas) de la voz en la comunicación presencial. Así, la persona que lea los textos escritos de esta forma, podrá imaginar las inflexiones en el tono de voz de la persona que escribió el mensaje, o en su caso, visualizar mentalmente los sonidos que este arrastre de vocablos ocasiona.

Continuando con los parámetros utilizados en las expresiones vocales emocionales, el recurso de la intensificación de un carácter o símbolo es utilizado para subrayar la acción que este signo trasmite. De este modo, es frecuente encontrarse en la CMO exageraciones o repeticiones de los signos de puntuación y exclamación para acrecentar el énfasis emocional de lo expresado, sea éste positivo o negativo, o en el caso de los sig-

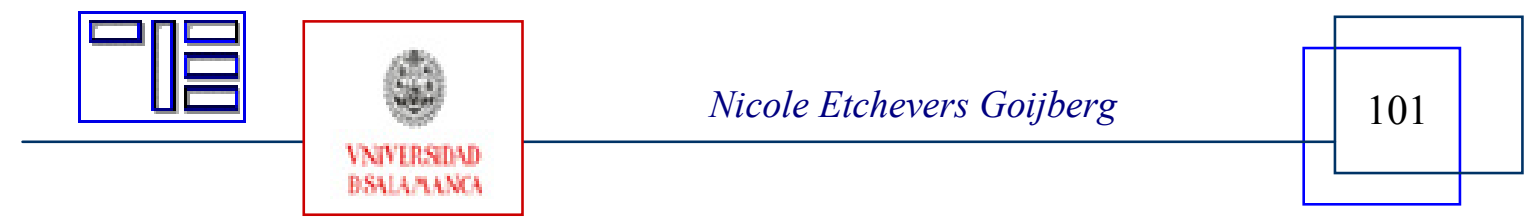


Revista Electrónica Teoría de la Educación.

Educación y Cultura en la Sociedad de la Información.

http://www.usal.es/teoriaeducacion

Vol. 7. No2. Diciembre 2006

nos de interrogación, para mostrar una pregunta, sorpresa, duda o desconcierto más allá de lo normal.

Derivándose también de lo anterior, el uso de los puntos suspensivos en la escritura de la CMO denota un silencio o pausa por parte del usuario, infiriéndose que el sujeto en cuestión, en su estado offline presencial, no se comunica de forma apresurada ni monótona a la hora de expresarse sino todo lo contrario, tiende a una cierta vivacidad, dándose tiempos para respirar y variar las características de su expresión vocal. De esta forma, realizando la analogía del paralenguaje en la CMO e incorporando además las conclusiones del estudio de Addington (1968), se puede deducir que los hombres y mujeres que utilizan exageradamente los signos ortográficos en sus escritos de CMO (y en general, el lenguaje complementado propuesto) serían proclives a tener rasgos de personalidad relativamente definidos y similares entre ellos.

Siguiendo con la teoría expuesta, es posible percibir determinados estereotipos de personalidad al escuchar las señales vocales de otro sujeto. Así, las conclusiones de Addington demuestran que la personalidad masculina es percibida generalmente en términos de poder físico y emocional, mientras que la personalidad femenina lo es en términos de facultades sociales. Utilizando esta investigación como marco de referencia, se puede definir que el hecho de utilizar reiteradamente los puntos suspensivos (y demás signos ortográficos) en la escritura de la CMO puede inferir juicios estereotipados de personalidad. Aunque se entiende que sería impropio generalizar o agrupar a todos los sujetos que escriben con estas características dentro de un mismo estereotipo de personalidad, no se puede dejar de considerar los beneficios que esto podría aportar en la Educación Online, específicamente ayudando a los docentes que trabajan en línea para hacerse una idea predefinida de ciertos rasgos de personalidad de sus alumnos.

Los usuarios de la CMO han tenido que representar y traducir de diferentes modos el gran repertorio del paralenguaje con el que se cuenta en la realidad para expresar la mayoría de sus estados emocionales. Es decir, se ha tenido que encontrar la manera de expresar las caracterizaciones vocales como la risa, el llanto, la sorpresa, la rabia, el suspiro, el bostezo, el estornudo, el ronquido, etc. y las segregaciones vocales como por ejemplo, los "hum", "m-mhmm", "ah", "uh" y variaciones de este estilo. (Trager, 1958 cit. en Knapp, 1982). De ello nace, a su vez, la utilización generalizada de las onomatopeyas. Al igual que con otras alteraciones lingüísticas explicadas en estos párrafos, se puede inferir ciertos rasgos de personalidad de los usuarios de este fenómeno puesto que para encontrar la representación textual más adecuada a la expresión que desean trasmitir, estos sujetos deben contar con ciertas características personales como creatividad, espontaneidad e imaginación.

Por último, los acrónimos, son utilizados para expresar frases comunes del lenguaje coloquial o para representar acciones exactas con mayor precisión y rapidez a la hora de escribir. Generalmente estos acrónimos son utilizados en entornos cerrados (tipo Chats y MUD's) donde los usuarios tienen un conocimiento preestablecido de las reglas de

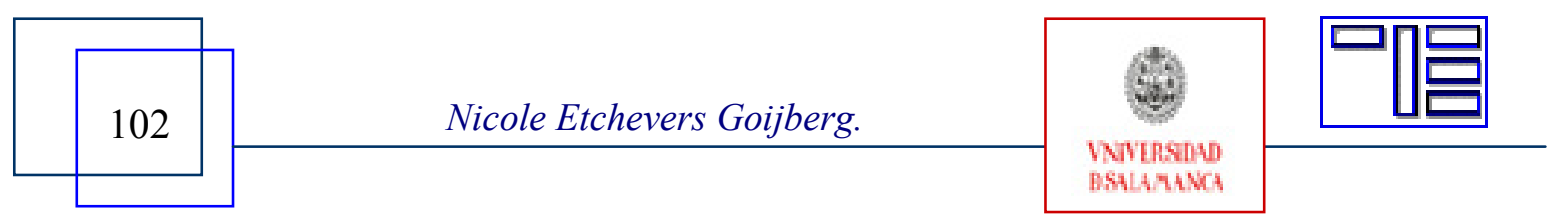


expresión usadas en aquel lugar. Y aunque es necesario hacer la aclaración que es más frecuente encontrar el uso de acrónimos en idioma inglés que en español, se ha considerado importante incorporarlos como parte del lenguaje complementado puesto que existen dentro de éstos, algunos que describen aspectos o estados emocionales de los usuarios. De esta forma, pueden verse representados algunas emociones básicas $(<b>$ para "blush" [sonrojarse]), sentimientos y tendencia a la acción ( $<$ lol $>$ para "laughing out loud" [riendo a carcajadas]; <rofl > para "rolling on floor laughing" [en el suelo de la risa]), resaltando el hecho que, además, dentro de estos acrónimos emocionales prácticamente todos pueden ser representados mediante el uso de emoticones gráficos. Por tanto, nuevamente, es posible considerar el uso de los acrónimos como referencia para forjarse a priori una idea de los rasgos de personalidad del usuario de CMO, puesto que en este caso, el hecho de acortar y abreviar palabras para acelerar la escritura revela una cierta tendencia a la velocidad, que, observado bajo la relación de las señales del paralenguaje y estereotipos de personalidad de Addington, determinarían a personas más extrovertidas y animadas.

\section{4.- CONCLUSIONES}

Los usuarios que se comunican mediante ordenador y a través de Internet, han debido encontrar originales maneras de representar lo mismo, o lo más similar posible, aquello que quisiesen comunicar si estuviesen en una comunicación cara a cara. Por tanto, y viéndose limitados en esta primera fase virtual exclusivamente a una expresión textual, aquella comunicación que no se puede expresar verbalmente (emociones, gestos, posturas, voz, etc.) debió encontrar otras salidas ajenas a las meras palabras. Así, nacieron nuevos código y lenguajes creados a partir de las mínimas herramientas textuales (y posteriormente gráficas) que ofrece por defecto cualquier ordenador.

En la investigación que lleva a cabo esta autora se denomina lenguaje complementado a aquella nueva forma de comunicación en la CMO que integra además de las palabras, una representación de las características no verbales y emocionales que suelen encontrarse en cualquier comunicación presencial. El término "complementado" se utiliza expresamente, puesto que implica tanto que el lenguaje hasta ahora utilizado en la CMO necesitaba ser "complementado" con algo más para ser eficaz e integral, como por otra parte, que este nuevo lenguaje creado se constituye internamente de diferentes componentes. Así, en este lenguaje complementado que complementa a la $\mathrm{CMO}$ es posible reconocer por tanto, dos aspectos básicos que contribuyen a que los usuarios de las TIC se comuniquen emocionalmente de una manera eficaz: (i) los emoticones y (ii) las alteraciones lingüísticas, con todos los aspectos que la integran (sustitución de consonantes complejas; abreviación y contracción de palabras; uso de mayúsculas; repetición de vocablos y signos de puntuación; onomatopeyas y acrónimos.

Si bien la CMO jamás reemplazará la riqueza de una comunicación cara a cara, este nuevo lenguaje complementado viene a reforzar una comunicación que en la mayoría de los casos se efectúa(ba) de manera estática y a-corporal, al menos que existiesen dispo-

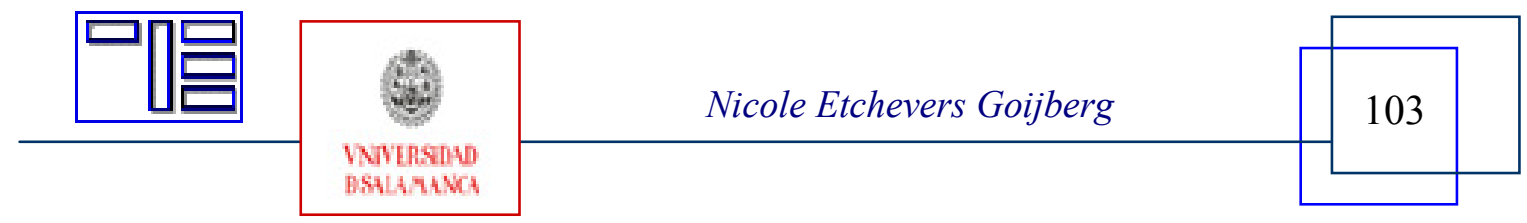


Revista Electrónica Teoría de la Educación.

Educación y Cultura en la Sociedad de la Información.

http://www.usal.es/teoriaeducacion

Vol. 7. No2. Diciembre 2006

sitivos externos como cámaras o aparatos de audio que los reforzaran. El lenguaje complementado, por tanto, podría concluirse que es una forma de representar, expresar e integrar por medio de texto y gráficos, aquello que el cuerpo y la comunicación no verbal realiza habitualmente en una comunicación presencial. Si bien con ello se logra que en la CMO sea posible mantener una comunicación emocional eficaz y al menos, por el momento, lo más cercano a la integralidad que una comunicación textual y virtual puede llegar a ser, una de las características más beneficiosas de contar con este tipo de lenguaje emocional integral en la CMO es que posibilita la opción de aprehender y conocer algunas características personales de los sujetos con los que se interactúa.

No está de más también comentar aquí que las alteraciones lingüísticas que abundan en la CMO, son materia de fuerte controversia en el mundo de la educación y han dado pie a muchos estudios sobre el efecto que las nuevas tecnologías están teniendo y tendrán a futuro, en el conocimiento y uso de la lengua. A pesar que muchos educadores aún consideran grave y perjudicial lo que sucede con el manejo de la lengua escrita en los entornos virtuales, en específico chats y mensajería instantánea, existen otros docentes que se muestran a favor del uso de las TIC por parte de los niños y jóvenes, puesto que aducen que ha fomentado, con creces, la forma de expresarse de las nuevas generaciones.

"Los educadores que están en contacto con chicos que hacen un uso intensivo de la mensajería instantánea y del e-mail, están viendo emerger una nueva generación de escritores adolescentes acicateados por una potestad tecnológica que ha multiplicado como nunca la potencia expresiva y permite que gente de todas las edades escriba más que nunca" (Piscitelli, 2004).

\section{5.- BIBLIOGRAFÍA}

ADDIGGTON, D. W. (1968): The Relationship of Selected Vocal Characteristics to Personality Perception, Speech Monographs, 35, 492-503.

AIMC - Asociación para la Investigación de los Medios de Comunicación ( $7^{\text {a }}$ Encuesta "Usos de la Red" 2005) Disponible en: http://www.aimc.es

ARANGO PINTO, L. G. (2004): Códigos de Comunicación para la interacción en el chat Comunicación presentada en II Congreso ONLINE del Observatorio para la CiberSociedad. Noviembre 2004. Disponible en:

http://www.cibersociedad.net/congres2004/index ca.html

BAKER, A. (2000): Two by Two in Cyberspace: Getting Together and Connecting Online, CyberPsychology \& Behavior, 3 (2), 237-242.

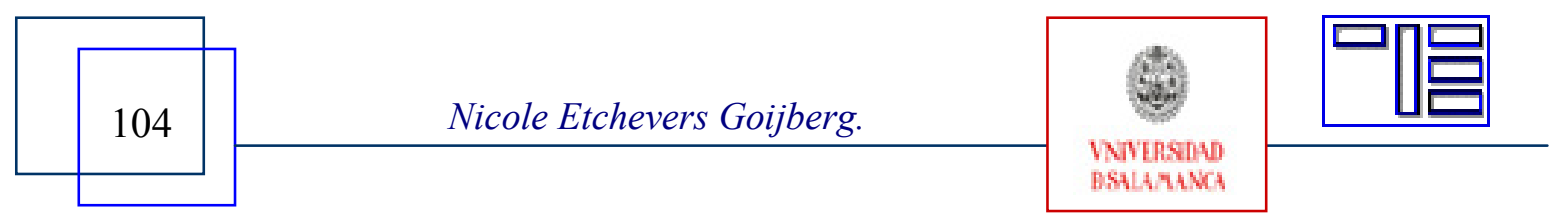


BESCÓS CALLEJA, V. (2002): El amor en la Red: del amor platónico al amor virtual. Artículo de Archivo del Observatorio para la CiberSociedad. Disponible en: http://www.cibersociedad.net

BONEVA, B.; KRAUT, R. y FROHLICH, D. (2000): Using e-mail for personal relationships. The Difference Gender Makes, American Behavioral Scientist, 45 (3), 530 549 .

CUMMINGS, J., BUTLER, B. y KRAUT, R. (2002): The Quality of Online Social Relationships, Communications of the $A C M, 45$ (7), 103-108. Disponible en: http://homenet.hcii.cs.cmu.edu/progress/cummings02-

QualityOfOnlineRelationships.pdf

DAVITZ, J.R. (1964): The Communication of Emotional Meaning. New York, McGraw Hill.

DE LA TORRE, S. (s.f.): Estrategias creativas para la educación emocional. Universidad de Barcelona. Disponible en:

http://www.iacat.com/webcientifica/Estrategiascreativas.htm

DEL BRUTTO, B. A. (1999): Situaciones amorosas. ¿Relaciones reales o virtuales? DOSSIER de KAIROS, 3 (4) 2do. Semestre.

DONN, J. y SHERMAN, R. (2002): Attitudes and Practices Regarding the Formation of Romantic Relationships on the Internet. CyberPsychology \& Behavior, 5 (2), 107-123. Disponible en: http://juno.ingentaselect.com

EKMAN, P. (2004): ¿Qué dice ese gesto? Barcelona, RBA Libros

EKMAN, P. y FRIESEN, W. V. (1969): The Repertoire of Nonverbal Behavior: Categories, Origins, Usage and Coding, Semiotica, 1, 49-98.

ETCHEVERS, N. (2006): El destape de las ciberrelaciones. Artículo de Divulgación. Disponible en: http://www.cibersociedad.net/recursos/art div.php?id=122

GIL, A., FELIU, J., RIVERO, I. y GIL, E. P. (2003): ¿Nuevas tecnologías de la información y la comunicación o nuevas tecnologías de relación? Niños, jóvenes y cultura digital UOC. Disponible en: http://www.uoc.edu/dt/20347/index.html

GORDO LÓPEZ, A. J. (2002): Función de las Nuevas Tecnologías en la Construcción de la Identidad: Una mirada cualitativa desde la E-moción y el Tacto, Revista Teknokultura, 2 Disponible en: http://teknokultura.rrp.upr.edu

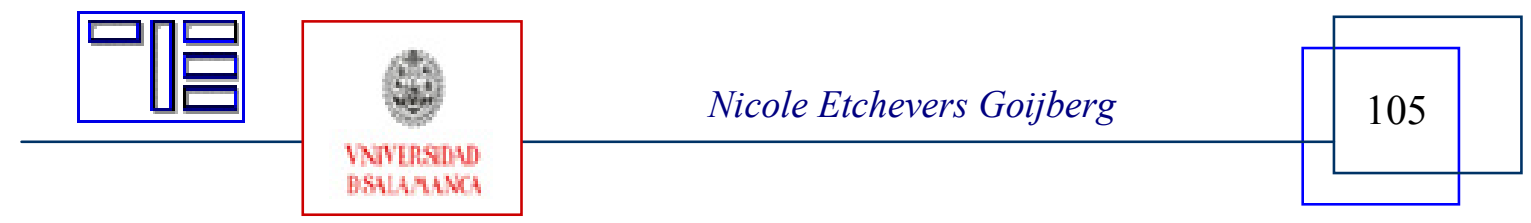


Revista Electrónica Teoría de la Educación.

Educación y Cultura en la Sociedad de la Información.

http://www.usal.es/teoriaeducacion

Vol. 7. No2. Diciembre 2006

KNAPP, M. (1982): La comunicación no verbal. El cuerpo y el entorno. Barcelona, Paidós.

KRAUT, R., MUKOPADHYAY, T., SZAZYPULA, J., KIESLER, S., y SCHERLIS, W. (2000): Information and Communication: alternative uses of the Internet in households, Information Systems Research 16, 287 - 363.

MAYANS I PLANELLS, J. (2001): Género Chat. O cómo la etnografía puso un pie en el ciberespacio. Barcelona, Gedisa.

MAYANS I PLANELLS, J. (2006): “El fenómeno MATCH”. Artículo de Divulgación. Disponible en: http://www.cibersociedad.net/recursos/art div.php?id=123

MCCOWN, J.; FISCHER, D.; PAGE, R. y HOMANT, M. (2001): Internet Relationships: People Who Meet People, CyberPsychology \& Behavior, 4 (5), 593-596.

PEW INTERNET AND AMERICAN LIFE PROJECT, (2000). Disponible en: http://www.pewinternet.org/reports

PISCITELLI, A. (2004): ¿Internet le hace bien o mal a la escritura? Veremos, veremos, veremos. Disponible en: http://weblog.educ.ar/educacion-tics/archives/001933.php

ROSENGREN, K. E. (2000): Communication. An Introduction. London, Sage.

SCHERER, K. y EKMAN, P. (Ed.) (1984): Approaches to Emotion. Hillsdale, New Jersey, Lawrence Erlbaum Associates, Inc.

SMITH, M. y KOLLOCK, P. (Ed.) (2003): Las comunidades en el Ciberespacio. Barcelona, UOC Colección Nuevas Tecnologías y Sociedad.

ROCO, G. (2001): Un estudio del establecimiento de relaciones íntimas mediadas por computador. El caso de IRC en Internet. Revista TEXTOS de la CiberSociedad, 1. Disponible en: http://www.cibersociedad.net

\section{Para citar este artículo puede utilizar la siguiente referencia:}

ETCHEVERS GOIJBERG, Nicole (2006): Los nuevos códigos de la comunicación emocional utilizados en Internet. En GARCÍA CARRASCO, Joaquín (Coord.) Estudio de los comportamientos emocionales en la red [monográfico en línea]. Revista electrónica Teoría de la Educación: Educación y Cultura en la sociedad de la información. Vol. 7, $\mathrm{n}^{\mathrm{o}}$ 2. Universidad de Salamanca. [Fecha de consulta: dd/mm/aaaa]. $<$ http://www.usal.es/ teoriaeducacion/rev_numero_07_02/n7_02_nicole_etchevers.pdf > ISSN 1138-9737

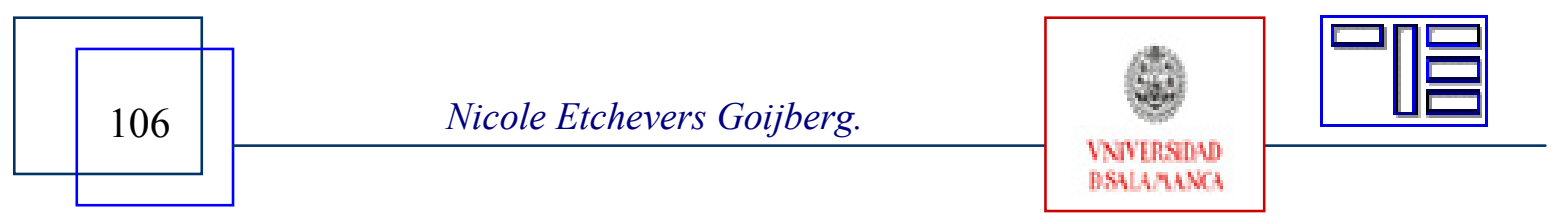

